\title{
CONCEPÇÕES DE ENFERMEIRAS SOBRE SEU TRABALHO NO MODELO CLÍNICO DE ATENÇÃO À SAÚDE ${ }^{1}$
}

\author{
NURSES' CONCEPTIONS ABOUT THEIR WORK IN THE CLINIC MODEL \\ ASSISTANCE \\ CONCEPCIÓN DE ENFERMERAS SOBRE SU TRABAJO EN EL MODELO \\ CLÍNICO PARA ATENCIÓN A LA SALUD
}
Maria Alice Dias da Silva Lima² Andréia da Silva Gustavo ${ }^{3}$ Débora Fernandes Coelho ${ }^{4}$ Úrsula Vogel Schmitz ${ }^{4}$ Raquel Borba Rosa

\begin{abstract}
RESUMO: O objetivo do estudo foi conhecer a concepção de enfermeiras sobre a ênfase das atividades que desenvolvem no processo de trabalho, no modelo clínico de atenção à saúde. Trata-se de uma pesquisa qualitativa, com abordagem dialética. Para compor a amostra foi sorteada uma enfermeira de cada unidade de internação de um hospital universitário de Porto Alegre/RS, totalizando 17 sujeitos. Os dados foram coletados através de entrevista semi-estruturada. Para a análise de dados procedeuse à classificação do material em estruturas de relevância, a partir das quais foram estabelecidas nove categorias. Os resultados evidenciaram que, embora haja uma predominância de atividades de gerenciamento do cuidado em relação às atividades assistenciais, as enfermeiras consideraram que essas dimensões são indissociáveis em seu trabalho. Concluiu-se que a relação entre assistir e gerenciar não é excludente e que o gerenciamento constitui-se em uma das dimensões do cuidado de enfermagem
\end{abstract}

PALAVRAS-CHAVE: trabalho da enfermeira, funções da enfermeira, papel da enfermeira

\section{INTRODUÇÃO}

A enfermagem brasileira sempre preocupou-se com o que as enfermeiras ${ }^{6}$ fazem nos serviços de saúde, o que pode ser constatado nos diversos estudos sobre suas funções, tanto na área hospitalar como na área de saúde pública (Trevizan, 1987; Balielo, 1981; Burlamaque, 1981; Adami, 1980). O predomínio dos aspectos gerenciais no trabalho que a enfermeira desenvolve na produção de cuidados de saúde no modelo clínico foi comprovado por Almeida (1991), Castellanos (1987), Kurcgant et al. (1994a, 1994b), Lima (1998).

Porém, para Kurcgant et al. (1994b), há uma ambigüidade em relação à função administrativa da enfermeira, tanto no nível teórico quanto no nível prático, sendo interpretada ora como administração da assistência, ora como administração do serviço. Consideram que

\footnotetext{
1 Pesquisa vinculada ao Núcleo de Estudos em Saúde Coletiva e Serviços de Saúde (NESCOSS) da Escola de Enfermagem da Universidade Federal do Rio Grande do Sul (UFRGS).

2 Professora Adjunta da Escola de Enfermagem da UFRGS. Doutora em Enfermagem pela Universidade de São Paulo.

${ }^{3}$ Enfermeira. Mestranda em Enfermagem pela UFRGS. Bolsista da CAPES.

${ }^{4}$ Acadêmica de Enfermagem da Universidade Federal do Rio Grande do Sul.

${ }^{5}$ Bolsista do Programa Institucional de Bolsas de Iniciação Científica - PIBIC CNPq/ UFRGS.

${ }^{6}$ Adotamos o substantivo feminino, de acordo com a designação cultural genérica utilizada para essa categoria profissional.
} 
essa ambigüidade decorre da falta de definição do que é a administração aplicada à enfermagem. Essa situação é reforçada pela formação, voltada, principalmente, para o cuidado direto, em contraposição às exigências da prática nas organizações de saúde. Para Lunardi Filho e Lunardi (1996), essas organizações obedecem à lógica capitalista, que requer uma enfermeira com competência técnica, científica e competência administrativa, indispensável para assumir a coordenação e o gerenciamento do trabalho coletivo.

Lima (1998), Lunardi Filho (1998), Lunardi Filho e Lunardi (1996), Trevizan (1987) mostram que as enfermeiras estão desempenhando, principalmente, a gerência dos serviços, o controle do material e do pessoal, em detrimento das atividades assistenciais, que são as preconizadas e transmitidas pelas escolas. A partir daí, questiona-se qual é a lógica de haver um ensino voltado a prestar cuidados a um único paciente, na maior parte dos cursos de graduação, prática essa muito distante do cotidiano da enfermeira.

Lima (1998) identificou que a enfermeira tem um papel coordenador nas atividades dos demais trabalhadores da equipe de saúde, envolvidos no cuidado ao paciente. Ela articula, supervisiona e controla ações que são desenvolvidas parceladamente pelos trabalhadores da saúde, tanto referentes ao pessoal de enfermagem como aos procedimentos voltados para o diagnóstico e tratamento. É a enfermeira que interliga o trabalho médico ao trabalho de enfermagem e estabelece a articulação da enfermagem com os demais trabalhos que compõem o processo de trabalho no hospital. O papel gerencial da enfermeira é reconhecido e valorizado pelos demais agentes da equipe de saúde, que apontam inúmeras atividades nas quais a presença da enfermeira se torna necessária e indispensável para garantir o desenvolvimento do trabalho coletivo.

Os resultados de Gaidzinski et al. (1998) evidenciam que, para as enfermeiras, a função administrativa é fundamental, pois assegura a organização do serviço e coordenação da equipe, visando a assistência adequada à clientela. Para Waldow (1998), em geral, as enfermeiras são educadas para administrar unidades, sendo que as escolas, apesar de enfatizarem a administração e o gerenciamento e fornecerem princípios administrativos para o desempenho dessa função, não oferecem o preparo necessário para uma atuação com segurança e autonomia.

A atividade gerencial da enfermeira é uma situação concreta na realidade histórica da enfermagem brasileira. Porém, a discussão sobre a finalidade de seu trabalho parece continuar existindo, tanto entre as enfermeiras que exercem atividades no âmbito da saúde como entre docentes de enfermagem e pesquisadores da área. Conforme Gaidzinski et al. (1998), a questão entre o cuidar e o administrar tem gerado polêmica. Rosa et al. (1989), Lunardi Filho e Lunardi (1996) consideram que a dicotomia entre a formação e a prática concreta é um dos motivos dessa controvérsia, a qual tem originado crise de identidade profissional, provocando a evasão de muitas enfermeiras para outras áreas.

Os estudos até aqui apresentados, em geral, partiram da análise das atividades executadas pela enfermeira, mostrando que a atividade gerencial é a que se sobressai. Apesar desses resultados, as enfermeiras idealizam as atividades assistenciais como aquelas que devem ser prioritárias em seu trabalho. Em função disso, utiliza-se, nesta investigação, a descrição que as enfermeiras fazem com relação às atividades por elas realizadas.

Com base na concepção de enfermeiras sobre seu cotidiano, em uma prática hospitalar concreta, procura-se compreender a relação dicotômica entre o cuidar e o gerenciar, na sua interpenetração como parte de um todo, chamado trabalho da enfermeira no hospital.

Para enfocar o problema apresentado, tem-se como objetivo: conhecer a concepção de enfermeiras sobre a ênfase das atividades que desenvolvem no processo de trabalho, no modelo clínico de atenção à saúde. 


\section{METODOLOGIA}

\section{CARACTERIZAÇÃO DO ESTUDO}

Utilizou-se a metodologia qualitativa para apreender a realidade concreta e compreender os fenômenos e processos sociais presentes no cotidiano da enfermeira, que influenciam diretamente na concepção sobre seu trabalho.

$\mathrm{Na}$ metodologia qualitativa, utilizou-se a abordagem dialética. Nessa perspectiva, foram abordadas as concepções das enfermeiras, procurando entender que as singularidades contêm determinações da totalidade ( Almeida e Rocha, 1997).

Caracteriza-se como um estudo exploratório-descritivo por objetivar a identificação de um fenômeno e o seu conhecimento nos limites de uma realidade específica. O estudo exploratório permite aprofundar o conhecimento em torno de um determinado problema (Triviños, 1987).

\section{COLETA DE DADOS}

A coleta de dados foi realizada em todas as unidades de internação do Hospital de Clínicas de Porto Alegre (HCPA): seis unidades clínicas, seis unidades cirúrgicas, uma unidade obstétrica, três unidades pediátricas e uma unidade psiquiátrica.

Para constituição da amostra, foi realizado um sorteio entre as enfermeiras que compõem o quadro funcional de cada unidade de internação, sendo excluídas as que trabalham somente nos finais de semana. Assim, a amostra foi composta por sujeitos dos turnos da manhã, da tarde e da noite, totalizando 17 enfermeiras.

A técnica utilizada para obtenção do material empírico foi a entrevista semi-estruturada, a qual privilegia a obtenção de informações através da fala individual, revelando condições estruturais, sistemas de valores, normas e símbolos (Minayo, 1994). Assim, a entrevista traduz a representação das enfermeiras e constitui-se em uma aproximação do trabalho que realizam no âmbito hospitalar.

Utilizou-se um roteiro contendo questões sobre as atividades que realizam nas unidades de internação, solicitando também a descrição de uma jornada de trabalho. O roteiro foi testado com duas enfermeiras de áreas não incluídas na amostra. Após esse teste piloto, as perguntas foram reformuladas para possibilitarem um maior entendimento pelas entrevistadas.

O material empírico foi coletado no período de janeiro a junho de 2000. As entrevistas foram agendadas com os sujeitos selecionados e realizadas pelas pesquisadoras, com o uso de gravador, sendo, posteriormente, transcritas na íntegra.

Os princípios éticos foram respeitados, protegendo os direitos dos indivíduos envolvidos, levando em consideração os aspectos apontados por Goldim (1997) e as questões éticas para pesquisas em enfermagem expressas por Polit e Hungler (1995).

Solicitou-se autorização à Comissão de Ética da Instituição. O consentimento das entrevistadas foi obtido através de um Termo de Consentimento Livre e Esclarecido, garantindo o anonimato e o direito de não participação em qualquer momento. Para preservar o anonimato, as entrevistadas foram identificadas por um código.

\section{ANÁLISE DOS DADOS}

Para a análise dos dados utilizou-se a fundamentação da perspectiva dialética, que permite contemplar os dados empíricos com suas particularidades, sem perder de vista a correlação com a perspectiva histórica e social. Conforme proposta de Minayo (1994), constituiu-se dos seguintes passos: ordenação dos dados, classificação dos dados e análise final. 
$\mathrm{Na}$ fase de ordenação, as fitas foram transcritas e as entrevistas foram identificadas com códigos, conforme a seqüência cronológica. Após, procedeu-se à classificação dos dados, através de leitura flutuante, para identificar idéias centrais e estruturas de relevância, nas quais alguns elementos que foram enfatizados nas entrevistas determinaram o confronto do material empírico com a fundamentação teórica.

Foram estabelecidas as seguintes estruturas de relevância: atividade administrativa, atividade assistencial, atividade de articulação, autoconcepção, ênfase, expectativa da instituição, expectativa dos outros profissionais, interdependência e atividades educacionais.

Para operacionalizar a separação dessas estruturas e seu reagrupamento, foi utilizado o software Ethnograph 5.0 (Qualis Research Associates), cujos recursos permitiram a codificação de segmentos do texto e seu agrupamento por temas, conforme classificações estabelecidas pelas pesquisadoras. Foi possível associar categorias e reagrupá-las, agilizando o processo de interpretação e construção teórica, segundo comenta Webb (1999).

Para realizar a análise final, procurou-se articular o material empírico e o referencial teórico, visando ultrapassar a simples descrição dos dados e tentando estabelecer relações que possibilitem novas explicações.

\section{DISCUSSÃO E INTERPRETAÇÃO DOS RESULTADOS}

A classificação em categorias permitiu visualizar as partes que compõem o trabalho da enfermeira. Entretanto, para discussão e interpretação dos dados, foi necessário recompor o todo para facilitar a compreensão da realidade apreendida. Por esse motivo, optou-se por apresentar os resultados de forma integrada, sem utilizar a divisão em categorias que resultaram do processo de análise, pois entende-se que as atividades desenvolvidas pela enfermeira, no processo de trabalho coletivo, não podem ser fragmentadas.

A análise dos resultados evidenciou que há uma predominância de atividades de gerenciamento do cuidado em relação às atividades assistenciais:

Eu sei que a gente acaba tendo um envolvimento muito grande com a questão burocrática, mas na minha percepção eu acho que o cuidado é muito importante; embora talvez eu faça isso muito também, às vezes a gente acaba ficando mais um gerenciador, do que próprio assistente.(ENF6)

Identificou-se que essa enfermeira considera que o paciente é a prioridade, atribuindo maior importância ao cuidado direto, o qual não tem sido a atividade predominante em seu trabalho e ainda está distante da realidade do cotidiano hospitalar. Parece ser uma situação idealizada, pois a seguir refere que é a atividade gerencial que predomina em seu trabalho. Apesar disso, algumas enfermeiras denotam pouca aceitação de sua função gerencial.

E eu gosto de trabalhar mais na assistência, mais no cuidado, né, de entrar no quarto, de fazer algum procedimento. A parte mais burocrática ela atrapalha um pouco. (ENF16)

(...) então a assistência, que devia ser o principal, fica em detrimento dessas outras coisas. (ENF17)

A descrição de um turno de trabalho da enfermeira denota a ênfase no papel de gerência que ela desempenha, confirmando os resultados dos estudos de Lima (1998), Gonçalves (1994) e Almeida (1991), que constataram que a enfermeira tem exercido, principalmente, atividades administrativas de controle e supervisão do processo de trabalho. 
(...) começo pela passagem de plantão, aí a gente tenta perguntar as dúvidas que tem nas atividades ou em algum acontecido, alguma medicação que ficou pendente ou que tem que ser feita, algum exame que tem que ser realizado, tento tirar essas dúvidas na passagem de plantão e, às vezes, as modificações na hora mesmo eu já tento passar pro auxiliar que tá ali. (ENF15)

As atividades de gerenciamento do cuidado foram relacionadas à supervisão e ao controle do processo de trabalho. Verificou-se que as atividades de coordenação, supervisão e controle dos agentes da equipe de enfermagem são destinadas às enfermeiras, conforme evidenciado a seguir:

Eu chego, primeiro recebo o plantão, vejo o que é prioridade em termos de cuidado ou pacientes que estão mais instáveis, dou uma checada nos pacientes que estão mais estáveis, aciono o plantão se necessário, vejo, então, alterações de medicações, chamar plantão, passar informação para o auxiliar, delegar tarefas com mais prioridade. (ENF6)

As enfermeiras citaram diversas situações nas quais elas supervisionam o trabalho, verificando se os auxiliares cuidam bem dos pacientes, se realizam as técnicas corretamente, se há exames para serem coletados.

(...) a gente faz a pesquisa de exames a serem coletados pelos pacientes (...) todos os exames a serem coletados pelo paciente, a gente faz uma pesquisa pelo computador e passa para os auxiliares fazerem essa coleta, a gente faz uma pesquisa também de exames a serem realizados, normalmente a enfermeira do turno que está saindo deixa todos os exames com preparo a ser realizado já prontos, com os preparos prontos e com os exames já marcados, que é marcado pela radiologia ou pelo setor onde vai fazer (...). (ENF4)

Essas situações coincidem com achados de estudo anterior de Lima (1998), no qual foi identificado que as enfermeiras, ao entrarem nas enfermarias, verificam como estão os pacientes ou certificam-se sobre a execução de tarefas e procedimentos, como forma de controlar o trabalho realizado pelos agentes de enfermagem.

Um outro detalhe é que durante a visitação de enfermagem a gente avalia se os equipos não estão vencidos, se tá tudo dentro da unidade de maneira adequada, se o soro tá dentro do gotejo adequado, então a gente faz toda uma avaliação dessa parte também. (ENF13)

A supervisão prioriza o que os funcionários fazem e sua atuação junto ao paciente. Portanto, sua concepção sobre as atividades de supervisão foi voltada para a vertente de fiscalização, com ênfase na verificação das atividades realizadas, correspondendo à caracterização de Almeida (1991) e de Lima (1998).

As enfermeiras articulam os trabalhos dos médicos e dos auxiliares de enfermagem, transmitindo aos auxiliares as solicitações ou ordens médicas:

(...) resolvo coisas com as equipes médicas, do soro que o paciente tá mantendo e não tá prescrito, do oxigênio que não está prescrito, eu vou atrás das equipes para resolver essa coisa da prescrição médica que eles deixaram de fazer ou dúvidas que a gente tem, ou medicação mal prescrita, que acontece com freqüência, então a gente vai atrás, resolve junto com a equipe. (...) oriento 
funcionários quanto à mudança de alguma prescrição médica, que tenha alterações, inclusão de medicação como aconteceu agora e daí oriento e esse funcionário vai já fazendo as coisas conforme ele é orientado. (ENF13)

As enfermeiras coordenam o trabalho que é realizado na unidade, denotando que, todo trabalho desempenhado coletivamente por divisão de funções, necessita de um profissional que cuide para que o conjunto se direcione de modo organizado para o produto, conforme apontado por Gonçalves (1994).

"Eu vejo muito que os outros pensam mais na enfermeira como gerente, assim, de uma unidade, quem comanda a unidade." (ENF2)

As atividades da enfermeira são muito interrompidas, pois ela é freqüentemente solicitada por outros profissionais, para dar informações sobre o paciente, ou para organizar a seqüência de realização de procedimentos aos quais o paciente precisa ser submetido. Ou, ainda, para atender outros tipos de solicitações que as fazem transitar, constantemente, pela unidade:

(...) te chamam pra tudo, é a limpeza, é a copa, é o seu doutor, são os funcionários, e é, não te dão chance mesmo de respirar, que nem eu digo, quando tu olha o relógio, "bah, onze horas, meu Deus! Ainda falta isso, isso e isso", vai, voa o tempo. (ENF5)

(...) mas a rotina, se tu for ver, na verdade não tem muito uma rotina, a gente não pode ligar muito porque tu tá aqui conversando, daqui a pouco alguém já chega e te chama. Se tu tá dentro de um quarto visitando o paciente, tu pára pra fazer aquilo, a gente não trabalha com... então é muito instável...te chamam e tu tem que ir. (ENF14)

Constatou-se que a enfermeira é responsável pela manutenção das condições necessárias à realização do trabalho coletivo, tais como recursos humanos e infra-estrutura.

É a parte que tu fica aqui no posto vendo escala, vendo hora-extra e vendo folga, e quem é que trabalha de tarde, quantas eu tenho, e se não tenho onde é que eu vou tirar, e liga pra fulana, a fulana vem, não pode, tu vai pra escala e aí eu remanejo, essa parte de ficar agilizando e vendo (...). (ENF5)

(...) se eu não manter a unidade com material, com pessoal, como é que eu vou dar uma boa assistência pros pacientes (...). (ENF17)

Confirmando os resultados encontrados por Lima (1998), identificou-se, na concepção que as enfermeiras têm sobre suas atividades, que elas exercem um papel articulador dos diversos trabalhos necessários aos cuidados envolvidos no processo terapêutico e que giram em torno do paciente. A enfermeira faz a articulação do trabalho de enfermagem com o médico e com outros setores do hospital.

(...) a gente visita os pacientes, encaminha todos os pacientes para os exames fora da unidade, radiografia, ecografia, qualquer tipo de exame ou avaliação em algum setor é sempre feito uma avaliação do enfermeiro (...). (ENF4)

Os residentes costumam perguntar para a enfermeira se os exames foram colhidos e 
encaminhados para o laboratório, ou se o paciente já foi chamado para realizar os procedimentos diagnósticos em outros setores do hospital:

Por exemplo, os médicos...Eu acho que na maioria os residentes que é com quem a gente tem contato, eles reconhecem muito o serviço da gente, tanto é que eles vêm na unidade e te questionam como é que tá o paciente, como é que foi o paciente. (ENF13)

Strauss et al. (1985), em pesquisa sobre a organização social do trabalho no hospital, abordam a necessidade de coordenação e articulação das atividades dos diversos agentes, para assegurar que os esforços coletivos da equipe não se tornem partes descontínuas e conflitivas. Entendem que o médico é a figura central no planejamento, mas é a enfermeira que conduz sua implementação, enquanto os outros trabalhadores de saúde, incluindo o pessoal de enfermagem e demais técnicos, executam as tarefas operacionais necessárias. Portanto, a enfermeira é o elemento chave para a articulação do trabalho, pois é ela quem organiza as diversas atividades de diagnóstico e terapêutica que giram em torno do paciente.

As atividades assistenciais, que são em menor proporção, caracterizam-se pela realização de etapas do processo de enfermagem, além da execução de procedimentos, na maioria aqueles que são exclusivos da enfermeira.

(...) a gente avalia se tem algum paciente mais crítico, a gente visita ele primeiro, vai dar uma avaliada no paciente, e a principio a primeira coisa que a gente faz é a avaliação de todos os pacientes, passa de quarto em quarto, faz visita, e vai avaliando dentro disso, das necessidades, qual o paciente que tem que ver alguma coisa (...) faz prescrições, evoluções de alguns, baixas também, agora mesmo eu tava terminando de fazer uma baixa, de noite tem muitas baixas, e é uma enfermeira só, então a gente perde no mínimo uma meia hora com cada paciente que baixa só na entrevista, no histórico. (ENF7)

Punção de veia com abocath, sonda nasoentérica, sonda vesical de demora, instalação e retirada de quimioterapia, instalação de NPT, coleta de sangue em pacientes do TMO autólogo que nós temos aqui na unidade, esses são os mais freqüentes...coleta de hemocultura, temos a infusão das células no TMO autólogo, nós entramos pois é a enfermeira que efetua isso. (ENF13)

Constatou-se, ainda, a interdependência do trabalho da enfermeira com o de outros profissionais, ou seja, a dependência de um trabalho ao do outro, tornando as ações em saúde complementares.

(...) o paciente tá lá fazendo uma bacteremia, se eu não disser pra equipe que o paciente tá com febre, tremor, cianose, se eu não coletar um sangue do paciente ou não identificar isso como importante, vai passar despercebido. É importante esse registro, exames de urina, isso é muito importante, isso faz parte do tratamento e do diagnóstico do paciente (...). (ENF14)

Conforme Lopes (1996), a assistência hospitalar caracteriza-se por relações de trabalho dinâmicas e interdependentes, com o reconhecimento dos espaços de saber e da ação de cada um. Lima (1998) considera que a interdependência entre os profissionais faz com que a autonomia de cada um seja relativa.

Frente à diversidade das atividades desenvolvidas pela enfermeira evidenciou-se, também, 
o papel educacional relacionado com acadêmicos de enfermagem e equipe, tendo em vista que é um hospital-escola:

(...) a gente também trabalha com alunos de enfermagem, (...) a gente orienta, a gente auxilia (...) a gente treina pessoal, tipo no período probatório do auxiliar de enfermagem ou do enfermeiro, tudo acompanhado, a gente tem uma ficha de avaliação do funcionários no período probatório (...). (ENF4)

Quanto à expectativa da instituição com relação ao trabalho dessa profissional, nota-se que o esperado é o gerenciamento da unidade:

Eu acho que a instituição espera que tu leve uma unidade, administre como tem que ser, eu acho que é o nosso papel de enfermeira (...). (ENF5)

A expectativa dos outros agentes concentra-se no papel da enfermeira como gerenciadora e articuladora das atividades dos diferentes profissionais em torno do paciente, necessários ao diagnóstico e à terapêutica, confirmando os resultados de Lima (1998).

(...) pra eles ser enfermeiro é ser a pessoa que organiza todo o contexto, várias pessoas comentaram isso, ou que o enfermeiro é o administrador que cada local deveria ter (...).

(ENF10)

A autoconcepção das enfermeiras está relacionada aos mitos da profissão, denotando responsabilidade por minimizar o sofrimento do paciente. Para elas, o trabalho de enfermagem tem características de doação, pois consideram que a enfermeira é "advogada do paciente", é como um "anjo", como "mãe".

Pra mim significa eu acho que dedicação por alguma coisa que eu gosto de fazer, acho que tem aquele lado que é a tua dedicação pro paciente, eu acho que isso, todo mundo diz, "ah, ser enfermeiro é tu ser o advogado do paciente", eu acho que tem um pouco desse lado (...). (ENF10)

(...) os pacientes geralmente dizem que a gente é anjo deles, que protege, que ajuda e eu me sinto um pouco isso aí, dessa maneira mesmo, não digo anjo, mas assim, de tentar dar o máximo de bem estar pra quem tá ali doente com vários problemas, mas a gente procura compensar um pouco as dificuldades que cada doença traz para os pacientes. (ENF7)

Acho que é doação. (...) De atenção. Porque na realidade, apesar disso que eu já relatei que a gente faz muitas atividades, tem o aspecto psicológico também envolvido ai, que eu acho importante. Então muitas vezes tu entra no quarto e o paciente tá bem fisicamente, mas psicologicamente ele não tá legal. (...) então escutar ou falar tu tem que te doar para eles, te desligar um pouco. Tu tá cheia de tarefas para fazer mas aquele paciente tá precisando de ti naquele momento, então tu tem que te doàr para ele. E eu acredito que a enfermagem é poder ajudar, poder fazer alguma coisa para melhorar a qualidade de vida dos pacientes. (ENF 13)

As enfermeiras, quando se referem ao seu trabalho, consideram que cuidar e gerenciar 
são atividades indissociáveis:

Eu acho que a gente tá aqui em função do paciente, tudo que tu faz em função do paciente é importante, se eu não for no quarto avaliar o meu paciente, ele pode ter daqui a pouco uma intercorrência que eu não identifiquei, então eu acho que tudo é importante, todas as atividades, assim como se a pessoa que tá aqui na medicação deixou faltar alguma coisa e a gente não se deu conta, o paciente não vai receber a medicação, acho que tudo que tu faz em prol do paciente eu acho que é o mais importante, não é uma nem duas atividades, é tudo que tu faz. (ENF10)

Olha, eu acho que não tem como dissociar, porque faz parte das tuas funções, não tem como tu sair disso, na verdade tu consegue fazer tudo junto, tu faz a parte assistencial, ai depois tu começa aquela parte administrativa, às vezes tu tem que largar assistencial e ir pra administrativa. (ENF5)

Tendo conhecimento das inúmeras atividades que constituem o trabalho da enfermeira no hospital, apreendidas nos dados empíricos, ratifica-se a compreensão de alguns autores (Bellato; Carvalho, 1998, Pereira; Silva, 1999) quanto à mulltidimensão desse trabalho, que atende a diferentes finalidades. Aponta-se, também, a necessidade de atenção para a complementaridade e interpenetração dessas dimensões, sem banalizar nenhuma delas, resultando na assistência ao paciente. Isso foi mencionado pelas enfermeiras, ao relatarem que todas as suas atividades, sejam elas administrativas, de cuidado, de articulação, educacionais, enfim, são em benefício do paciente. Dessa forma, pode-se afirmar que cuidar e gerenciar são indissociáveis e são dimensões do cuidado humano, se considerarmos que o paciente é o foco para o qual as ações são dirigidas, em qualquer uma dessas situações.

\section{CONSIDERAÇÕES FINAIS}

Os resultados evidenciaram que, embora haja uma predominância de atividades de gerenciamento do cuidado em relação às atividades assistenciais, as enfermeiras consideraram que essas dimensões são indissociáveis em seu trabalho.

O gerenciamento é uma dimensão do cuidado de enfermagem e, através dele, a enfermeira busca o suporte necessário para que se alcance o produto final do processo de trabalho no modelo clínico, a assistência ao paciente. Contudo, para que isso ocorra, é importante a articulação das ações dos diversos profissionais da equipe de saúde no hospital. Entende-se esse processo como um sistema dinâmico, em transformação, cujas atividades ocorrem de forma interdependente, ficando evidente que a complementaridade dessas ações está diretamente relacionada à articulação entre os profissionais, bem como à reciprocidade de cada um no processo.

Os resultados da investigação trazem à tona uma discussão passível de contribuir para o ensino e a prática assistencial de enfermagem nos serviços de saúde, permitindo compreender conflitos e indefinições referentes ao trabalho da enfermeira na área hospitalar, em sua inserção no trabalho coletivo na área de saúde.

Conclui-se que a relação entre assistir e gerenciar não é excludente e que o gerenciamento constitui-se em uma das dimensões do cuidado de enfermagem. 
ABSTRACT: The aim of this study was to know the nurses' conception about the emphasis of the activities that they develop in the work process, in the clinic model assistance. This study is a qualitative research, with dialetic approach. To form the sample one nurse was chosen in each of the 17 units of a university hospital in Porto Alegre/RS. The data were collected through semi-structured interview. To analize the data the material was classified in relevant structures, from which nine categories were established. The results showed that, although there is a prevalence of management activities of the care concerning the assistance activities, the nurses considered that these dimensions are indissociable in their work. It was concluded that the relation between assisting and managing are not excluding and that the management is one of the dimensions of nursing care.

KEYWORDS: nurse's work, nurse's functions, nurse's role

RESUMEN: El objetivo del estudio fue conocer a la concepción de enfermeras sobre el énfasis de las actividades las cuelas desarollan en el proceso de trabajo, en el modelo clínico para la atención a la salud. Es una pesquisa cualitativa com un abordaje dialéctica. A fin de componer a la muestra fue sorteada una enfermera de sendas unidades de internación de un hospital universitário de Porto Alegre/RS, totalizando 17 sujetos. Los datos fueron colectados través una entrevista semi-estructurada. Para el análisis de los datos se há clasificado los materiais en estructuras de mayores importancias, desde las cuales fueron determinadas nueve categorías. Los resultados evidenciaron que, aunque haia predominancia en las actividades de la gerencia del cuidado relacionado a las actividades asistenciales, las enfermeras consideraron que estas dimensiones son inseparables en su trabajo. Han concluido que las relaciones entre asistir y gerenciar no pueden excluirse y que el gerenciamiento es una de las dimensiones del cuidado de enfermería.

PALABRAS CLAVE: trabajo de la enfermera, funciones de la enfermera, papel de la enfermera

\section{REFERÊNCIAS BIBLIOGRÁFICAS}

ADAMI, N. P. A função assistencial exercida pela enfermeira nos Centros de Saúde da Secretaria de Estado da Saúde de São Paulo. Revista da Escola de Enfermagem da USP, São Paulo, v.14, n.1, p. $63-72,1980$

ALMEIDA, M.C.P. de. O trabalho de enfermagem e sua articulação com o processo de trabalho em saúde coletiva - rede básica de saúde em Ribeirão Preto. 1991. 297p. Tese (Livre Docência) Escola de Enfermagem de Ribeirão Preto, Universidade de São Paulo, Ribeirão Preto.

ALMEIDA, M.C.P. de; ROCHA, S.M.M( Orgs.). O trabalho de enfermagem. São Paulo: Cortez, 1997.

BALIELO, V. Análise das atividades do pessoal de enfermagem de um hospital-escola. 1981, 107p Dissertação (Mestrado) - Programa de Pós-graduação em Enfermagem, Universidade Federal do Rio Grande do Sul, Porto Alegre.

BELLATO, R. ; CARVALHO, E. C. Insignificâncias essenciais (a busca pelo reencantamento no quotidiano hospitalar). Cuiabá: EdUFMT, 1998.

BURLAMAQUE, C.S. Estudo do desempenho do enfermeiro de um hospital de ensino em nível de unidade de internação. Porto Alegre, 1981. 91p. Dissertação (Mestrado) - Programa de Pós-graduação em Enfermagem, Universidade Federal do Rio Grande do Sul. 
CASTELLANOS, B.E.P. O trabalho do enfermeiro. A procura e o encontro de um caminho para o seu estudo: da abordagem mecânico-funcionalista à pesquisa emancipatória.1987.442p. Tese (Doutorado) - Escola de Enfermagem da Universidade de São Paulo, São Paulo.

GAIDZINSKI, R.R; LEITE, M.M.J.; TAKAHASHI, R.T. O ensino da administração em enfermagem: percepção diante da vivência profissional. Revista Escola de Enfermagem da USP, v.32, n.1, p.42-51, abr. 1998

GOLDIM, J.R. Manual de iniciação à pesquisa em saúde. Porto alegre: Dacasa, 1997.

GONÇALVES, R.B.M. Tecnologia e organização social das práticas de saúde: características tecnológicas do processo de trabalho na rede estadual de centros de saúde de São Paulo. São Paulo, Hucitec - Abrasco, 1994

KURCGANT, P.; LEITE, M.M.; GAIDZINSKI, R.R.; PERES, H.H.C. O significado da administração aplicada à enfermagem segundo a opinião de graduandas. Rev. ESC. Enf. USP, v.28, n.1, p.1526, abr. 1994a

KURCGANT, P.; LEITE, M.M.; GAIDZINSKI, R.R.; PERES, H.H.C. O significado da administração aplicada à-enfermagem segundo a opinião de graduandas: parte II. Rev. Esc. Enf. USP, v.28, n.2, p.147-55, ago. 1994b.

LIMA, M.A.D. da S. O trabalho de enfermagem na produção de cuidados de saúde no modelo clínico, 1998. 216 p. Tese (Doutorado) - Escola de Enfermagem de Ribeirão Preto, Universidade de São Paulo, Ribeirão Preto.

LOPES. M. J. M. Poder, interdependência e complementaridade no trabalho hospitalar: uma análise a partir da enfermagem. Saúde, Porto Alegre, v.1, p.43-50, 1996.

LUNARDI FILHO, W. D.; LUNARDI, V. L. Uma nova abordagem no ensino de enfermagem e de administração em enfermagem como estratégia de (re)orientação da prática profissional do enfermeiro. Rev. Texto e Contexto UFSC, v. 5, n. 2, p.20-34, jul./dez. 1996.

LUNARDI FILHO, W. D. O mito da subalternidade do trabalho da enfermagem à medicina, 1998, 343 p. Tese (doutorado) Programa de Pós-Graduação em Enfermagem, Universidade Federal de Santa Catarina, Florianópolis.

MINAYO, M.C. de S. O desafio do conhecimento: pesquisa qualitativa em saúde. 3.ed. São Paulo: Hucitec, 1994.

PEREIRA, W. R.; SILVA, G. B. da. Tão longe, tão perto: mulher, trabalho, afetividade e poder. Cuiabá: EdUFMT, 1999.

POLIT, D.F.; HUNGLER, B.P. Fundamentos da pesquisa em enfermagem. 3.ed. Porto Alegre: Artes Médicas, 1995.

ROSA,M. T. L. da; RODRIGUES, A. M.; CASTELLANOS, B. E.; ALMEIDA, M. C. P. de; et al. O desenvolvimento técnico-científico da enfermagem - uma aproximação com instrumentos de trabalho. In: CONGRESSO BRASILEIRO DE ENFERMAGEM, 41.,Florianópolis, 1989. Anais... Florianópolis: ABEn, 1989. p. 97-126.

STRAUSS, A.; FAGERHAUGH, S.; SUCZEK, B.; WIENER, C. Social organization of medical work. Chicago: The University of Chicago Press, 1985.

TREVIZAN, M.A. A função administrativa do enfermeiro no contexto da burocratização hospitalar. Revista Brasileira de Enfermagem, v.40, n.4, p. 204-209, out./dez., 1987. 
TRIVIÑOS, A.N.S. Introdução à pesquisa em ciências sociais: a pesquisa qualitativa em educação. São Paulo: Atlas, 1995.

WALDOW, V. R. Cuidado humano: o resgate necessário. Porto Alegre: Sagra Luzato, 1998. Cap.6, p.161-198: As demais dimensões e espaços do cuidado humano na enfermagem.

WEBB, C. Analysing qualitative data: computerized and other approaches. Journal of Advanced Nursing, v.29, n.2, p. 323-330, Feb./1999. 